\title{
Materials for energy harvesting, conversion and storage
}

Coupling between materials and energy occurs in our environment every day at a visible scale. It affects society in space and time through returns from each generation's experiences.

Each energy strategy must consider the local or regional specificities, including the availability of resources, the effectiveness of the systems and the user's profiles. An energy strategy cannot be successful without considering the materials control [1] responding to production constraints, security, and recycling. This coupling contains many open problems, whose solutions could be technologically groundbreaking.

The energy sector demands new materials and this leads us to consider new uses for existing materials. One may take the example of materials such as wood or steel which are not especially new, but which must be used in an original fashion in the buildings of the future. The same is true for nanomaterials, for example nanotubes $[2,3]$ or solar components [4] that stimulate research in applied physics.

Beyond this aspect, the coupling between materials and energy [5] draws from basic disciplines such as the mathematical and physical sciences. If mathematics advocates the virtues of measurement, physics looks more specifically to energy and materials.

Physics sees energy as the ability of a system to interact with its environment. This manifests itself in producing movement, radiation or heat. Physics looks at materials as an arrangement of matter aimed at obtaining the desired properties necessary for specific applications. Human history considers the relationship of humans with both materials and energy. This duality constitutes the basis of any progress, from the development of humanity into its environment to its print on the environment.

This special issue is a snapshot of some advances at the materials/energy interface. It also aims to stimulate the curiosity of young researchers to take advantage from knowledge learned in universities and academic institutions, but also from their own observations on life and the universe.

R. Bennacer

Université Paris Saclay, ENS Cachan, France

M. El Ganaoui

Université de Lorraine, France

J.M. Nunzi

Queens University, Kingston, Ontario, Canada 
Le couplage matériaux et énergie a des manifestations évidentes sur notre environnement. Celles-ci deviennent visibles à l'échelle sociétale avec des retours générationnels.

Chacune des stratégies énergétiques devra puiser ses facteurs de succès des spécificités locales ou régionales selon la disponibilité des ressources, l'efficacité des systèmes et le comportement des usagers. Le succès d'une stratégie énergétique ne peut se faire sans considérer la maîtrise des matériaux [1] répondant à des contraintes de production, de sûreté, et de recyclage. Cette maîtrise nécessite elle-même des ressources énergétiques non toujours disponibles ou mobilisables. Ce couplage contient par nature des problèmes ouverts potentiellement intéressants pour obtenir des solutions technologiques de rupture.

Le secteur de l'énergie est demandeur de nouveaux matériaux, cela nous amène à envisager les matériaux sous l'angle de leurs propriétés nécessitant la mise en œuvre de moyens de physique originaux. On pourra prendre l'exemple de matériaux comme le bois ou l'acier qui ne sont pas spécialement nouveaux, mais dont la mise en œuvre dans le bâtiment du futur nécessite le développement et la mise en œuvre d'une physique originale. Il en va de même pour les nanomatériaux, nanotubes [2,3] par exemple ou les composants solaires [4] qui stimulent la recherche en physique appliquée.

Au-delà de cet aspect, le couplage matériaux-énergie [5], devenu visible, a bénéficié longtemps et largement des disciplines fondamentales telles que les sciences mathématiques et physiques. Si les mathématiques prônent par excellence les vertus de la mesure, la physique, elle, regarde de manière particulière l'énergie et les matériaux.

La physique voit l'énergie comme la capacité d'un système à interagir avec son environnement et à se manifester en produisant mouvement, rayonnement ou chaleur. La physique regarde les matériaux comme l'arrangement de matière permettant d'obtenir à souhait des propriétés voulues pour des applications spécifiques. L'histoire de l'homme peut se lire par son rapport aux matériaux mais aussi à l'énergie. Cette dualité constitue le socle du progrès, de l'emprise de l'homme sur son milieu mais aussi de son empreinte sur son environnement.

Cette édition spéciale se veut une image instantanée de certaines avancées à l'interface matériaux/énergie. Elle vise également à stimuler la curiosité des jeunes chercheurs pour profiter de la connaissance acquise dans les universités et les établissements scolaires, mais également de leur propre regard sur la vie et l'univers.

R. Bennacer

Université Paris Saclay, ENS Cachan, France

M. El Ganaoui

Université de Lorraine, France

J.M. Nunzi

Queens University, Kingston, Ontario, Canada.

\section{References}

1. M. El Ganaoui, R. Prud'homme, R. Bennacer, Guest Editors. C. R. Mecanique (Acad. Sciences), 335, 110 (2007)

2. A.B. Dalton, J.N. Coleman, M. in het Panhuis, B. McCarthy, A. Drury, W.J. Blau, B. Paci, J.M. Nunzi, H.J. Byrne, J. Photochem. Photobiol. A 144, 31 (2001)

3. H. Derbal-Habak, C. Bergeret, J. Cousseau, J.M. Nunzi, Sol. Energy Mater. Sol. Cells 95, S53 (2011)

4. J. Baffreau, S. Leroy-Lhez, H. Derbal, A.R. Inigo, J.M. Nunzi, M.M. Groeneveld, R.M. Williams, P. Hudhomme, Eur. Phys. J. Appl. Phys. 36, 301 (2006)

5. R. Bennacer, M. El Ganaoui, A. Mimet, Eds., in Actes Int. Conf. On Materials and Energy 2015, Tétouan, Morocco, p. 240, DOI: $10.13140 / \mathrm{RG} 2.1 .4654 .2240$ 